\title{
Profile of IHC Marker in Lung Tumours
}

\author{
Snigdha Petwal ${ }^{1}$, Aparna Bhardwaj ${ }^{1 *}$, Rajnish Kumar ${ }^{1}$, Sanjay Kaushik ${ }^{1}$, Sanjeev Kishore ${ }^{2}$ \\ ${ }^{1}$ Department of pathology, SGRR Institute of Medical \& Health Sciences, Patel Nagar, Dehradun, India \\ ${ }^{2}$ Department of Pathology, All India Institute of Medical science, Rishikesh, India
}

\begin{abstract}
Background: Lung biopsy is crucial in histopathological subtyping and classification. However, making an accurate diagnosis in small biopsies can be challenging. This study was aimed to distinguish and classify lung tumors employing immunohistochemical markers TTF1(thyroid transcription factor 1), Cytokeratin 5/6, Cytokeratin 20, AE1/3. Subsequently prognostic value of different histological types of lung tumours was established employing Ki-67.
\end{abstract}

Material \& Methods: A total of 52 clinically diagnosed cases of lung carcinoma over a period of two years were included in our study. Of these 2 cases were excluded from the study as they showed granulomas on histopathology. All the cases after being diagnosed on H\&E were subjected to Immuohistochemistry (IHC).

Statistical Analysis: The statistical analysis was done using SPSS for Windows 15.0 program. Specificity, sensitivity, positive predictive value, negative predictive value of all these IHC markers was statistically evaluated.

Results: Of 29 cases diagnosed as Squamous cell carcinoma (SCC) on H\&E, 26 were CK5/6 positive on IHC. Of 4 cases diagnosed as Primary Adenocarcinoma (ADC) on H\&E, 3 were TTF-1 positive. Of 4 cases diagnosed as poorly differentiated carcinoma on H\&E, 1 case was diagnosed as SCC and 2 cases as Metastatic ADC on employing IHC markers. Ki-67 was high in $82.8 \%$ cases of SCC and in $50 \%$ cases of ADC. The sensitivity and specificity of CK5/6 and TTF-1 was $100 \%, 57.1 \%$ and $100 \%, 96.7 \%$ respectively.

Conclusion: Employing IHC markers either singly or in a panel is a useful adjunct to morphological features and clinical parameter in the diagnosis and management of lung tumour.

\section{Keywords: Adenocarcinoma, CK5/6, Immunohistochemistry, Squamous cell carcinoma, TTF-1}

\section{Introduction}

Lung cancer is the leading cause of cancer related mortality worldwide. ${ }^{[1,2]}$ In India, lung cancer constitutes $6.9 \%$ of all new cancer cases and $9.3 \%$ of all cancer related deaths in both sexes ${ }^{[3]}$ Primary lung carcinomas have been divided into small cell lung carcinomas (SCLC) and non-small cell lung carcinomas (NSCLC). The main histologic types of NSCLC are adenocarcinomas (50-70\%), squmous cell carcinoma $(20-30 \%)$ and large celll carcinoma $(<10 \%){ }^{[4,5]}$

Majority of non-small cell lung carcinomas (NSCLC) can be diagnosed and further subtyped employing hematoxylin and eosin (H\&E) stains. However, morphological recognition of poorly differentiated tumours may be difficult, especially in smalll biopsies. ${ }^{[6]}$ To accurately diagnose these tumours there is an increasing demand of ancillary techniques such as IHC. Furthermore, IHC plays an important role in distinguishing metastatic lesion from primary lung carcinomas. ${ }^{[7]}$ Because of underlying difficulties to rely on single IHC marker, a panel of markers is suggested to improve sensitivity and specficity.
In squamous cell carcinoma p63 and Cytokeratin 5/6 are commonly used markers while TTF-1 and Napsin A have been proven to stain majority of lung adenocarcinomas. ${ }^{[8,}$ 9] Thus, this study was undertaken with an aim to identify a panel of IHC markers that can accurately distinguish Squamous cell carcinoma from Adenocarcinoma, tumours of epithelial origin from lymphoid origin and primary lung adenocarcinoma from metastatic adenocarcinoma.

\section{Materials and Methods}

This study was carried out in the Department of Pathologyin a tertiary care hospital in Uttarakhand. Approval of the study was taken from the institutional ethical committee before commencing the study.

It was a prospective study. All cases that were clinically diagnosed as lung tumour during a period of 20 months (October2015- July 2017) were included in the study. Total 52 clinically diagnosed cases of lung tumours were included in this study. Relevant history, clinical findings and radiological reports were recorded on the designated pre-tested proforma. 
Tissue samples were received as bronchial or pleural biopsies in $10 \%$ formalin. Grossing was done as per the protocol of histopathology laboratory. All the formalin fixed tissues were processed in automatic tissue (Shandon Citadel 2000). The blocks were made from processed tissue using Embedding station (Leica EG1150h+C).

Sections of $3-5 \mu$ were made using microtome. They were stained by haematoxylin and eosin, and mounted in DPX. The sections were studied using light microscope and detailed histopatholgy report was done by consultant histopathologist.

Immunostaining: For IHC, additional 5 sections of 2-4 microns from the above paraffin embedded blocks were made and taken on poly-L lysine coated slides. They were subjected to immunohistochemistry employing TTF-1 for Adencarcinoma, CK5/6 for SCC, CK20 to differentiate primary lung adenocarcinoma from metastatic Adenocarcinoma and immuno-staining for pan-cytokeratin, AE1/AE3 to demonstrates that the tumor is a carcinoma rather than a lymphoid tumor using primary antibodies DO7 (1/50 Dako) and Mib-1 (1/50 Novocastra). Sections were incubated with the secondary biotinylated antibody and avidin - biotin peroxidase complexes for 30 minutes. Reaction products were revealed with diminobenzidine (DAB) as the chromogen and sections were counterstained with Harri's hematoxylin to enhance nuclear detection.

Sections of normal thyroid tissue that was detected for TTF-1 and achronic tonsillitis that was detected for ki67 was used as positive controls. Sections of normal skin tissue that was detected for CK5/6, CK20 and AE1/3 was used as positive control. For negative controls, primary antibody was substituted with phosphate buffer saline (PBS) in duplicate sections.

Interpretation: The staining intensity and area of sections were evaluated under low magnification(X 20). Histologic $(\mathrm{H})$ scores were conducted following the recognised method for CK5/6, AE1/3, TTF-1 and CK20. A 4 level scoring for intensity was used ( 0 negative; $1+$, weak; $2+$ moderate; $3+$ strong). The expression intensity (0-300) was determined by multiplying the scores by the percentage of positive area $(0-100 \%)$. Sections with $\mathrm{H}$ score of $>150$ were considered to show a high expression, and those with $\mathrm{H}$ scores of $\leq 150$ were considered to show a low expression. ${ }^{[10]}$

For calculating the ki- index, the entire section was screened to find the region with maximum number of tumour cells showing positivity. Percentage of tumour cells that were positive was calculated by counting atleast 100 cells. The immunohistochemical results were interpretated according to the intensity of immunoreactive product seen in the nuclei of tumour cells.
Statistical analysis: The statistical analysis was done using SPSS for Windows 15.0 program. The sensitivity, specificity, PPV, NPV of markers CK5/6, TTF-1, CK20, AE1/3 for all the cases was calculated using Galan \& Gambino Method. Fisher's Extract Test was performed to compare categorical variables with status of $\mathrm{CK} 5 / 6$ and TTF-1 expression. A p value of $<0.05$ was accepted as statistically significant. ${ }^{[11]}$

\section{Results}

Of all the 52 cases that were clinically diagnosed as lung carcinomas, the $\mathrm{M}: \mathrm{F}$ ratio was $4.6: 1$ with maximum number of cases seen in the age group 61-80 years $(60 \%)$. The commonest presenting symptom in all these cases was fever with cough $(40 \%, 20$ cases $)$. Of all the 52 cases, a histopathologic diagnosis could be made in 42 cases $(80.8 \%)$. Of the remaining 10 cases, 4 cases were diagnosed as suspicious of malignancy with no confirmed histopathological category and 6 cases were grouped in the category of others. Of these 6 cases, 4 cases showed epithelial dysplasia and were included in the study while the other 2 cases showed necrotising granulomas on histopathology and were excluded from the study.

Histologic correlation of 50 cases of lung tumours included in the study with distribution pattern of all the IHC markers was done. (Table1)

Out of 29 cases of Squamous cell carcinoma diagnosed on H\&E (fig.1A), 26 cases (89.7\%) were positive for CK5/6 thus confirmed as squamous cell carcinoma (Fig.1B,C). All the 29 cases were negative for TTF-1 \& CK20. It was observed that out of 29 cases of SCC, 24 cases $(82.8 \%)$ showed high ki-67 index(1D). Out of 5 cases adencarcinoma diagnosed on H\&E, 4 cases $(80 \%)$ were positive for TTF-1 and one case $(20 \%)$ was positive for CK20. All the 5 cases were negative for $\mathrm{CK} 5 / 6$ but were positive for $\mathrm{AE} 1 / 3$. Hence, out of 5 cases diagnosed as Adenocarcinoma on H\&E, 4 cases were confirmed as primary adenocarcinoma while 1 case was confirmed as metastatic adenocarcinoma on IHC. A high Ki67 index was observed in 3 cases (60\%) out of 5 cases of Adenocarcinoma.

Out of 4 cases diagnosed as poorly differentiated carcinoma on H\&E (Fig.2A), all the 4 cases were confirmed of epithelial origin as they were positive for AE1/3. One case $(25 \%)$ exhibited positivity for CK $5 / 6$ hence confirmed as Squamous cell carcinoma and 2 cases exhibited positivity for CK20 thus were confirmed as metastatic adenocarcinoma (Fig 2B,C). None of the cases were positive for TTF-1(Fig. 2D). A high expression of ki67 (50\%) was seen in 2 cases out of 4 cases. 
Out of 4 cases diagnosed as Small cell carcinoma on H\&E, 2 cases $(50 \%)$ were positive for AE $1 / 3$ thus confirming the epithelial origin. Of these, 1 case showed positive expression for TTF-1 and was confirmed as Small cell carcinoma(Fig. 3A,B), None of the case exhibited a positive expression for either CK5/6 or CK20. Ki67 expression was high only in 2 cases.

On histopathology, 4 cases were categorised as Suggestive/ Suspicious of malignancy. Of these, 3 cases were positive for AE $1 / 3$ thus confirming the epithelial origin of tumour. Out of these 3 cases, 2 cases $(50 \%)$ exhibited a positive expression for CK5/6 thus confirming it to be Squamous Cell carcinoma (Fig.3C,D) and 1 showed a positive expression for TTF-1 thus confirming a diagnosis of Adenocarcinoma. One case showed no positive expression for either markers. A high expression of Ki67 was seen only in 1 case $(>25 \%)$.

Four cases showing epithelial dysplasia with atypical cells were grouped in the category of Others. Of these 4 cases, only 1 case $(25 \%)$ exhibited a positive expression for AE $1 / 3$ and CK5/6 and was labelled as Squamous Cell Carcinoma. The remaining 3 cases in this category were negative for all the markers and a repeat biopsy from representative area was asked on account of high degree of suspicion.

For Squamous cell carcinoma, Sensitivity, Specificity, PPV, NPV and accuracy for CK5/6 was $86.6 \%, 85 \%, 89.6 \%$, $81.0 \%$ and $69 \%$ respectively. Immunostaining results for sensitivity, specificity, PPV, NPV and Accuracy for TTF1 in Adenocarcinoma were 50\%, 97.7\%, 75\%, 93.5\% and $92.0 \%$ respectively. The overall diagnostic Accuracy of CK5/6, TTF-1 AE1/3\&CK20 was $60 \%, 92 \%, 82 \%$ and $92 \%$ respectively (Table 2). H score was calculated for all the markers (Table 3).

\section{Discussion}

The present study was conducted on bronchial and or pleural with regards to ability to diagnose and differentiate nonsquamous and squamous cell carcinomas. Differentiating

Table 1: Showing distribution pattern of all the IHC markers in all the 50 cases of lung tumors.

\begin{tabular}{|l|c|c|c|c|c|}
\hline Typing & CK5/6 & TTF-1 & AE1/3 & CK20 & Ki67(>20\%) \\
\hline SCC & $26(89.7 \%)$ & 0 & $29(100 \%)$ & 0 & $24(82.8 \%)$ \\
\hline ADC & 0 & $4(80 \%)$ & $5(100 \%)$ & $1(20 \%)$ & $3(60 \%)$ \\
\hline Poorly differentiated carcinoma & $1(25 \%)$ & 0 & $4(100 \%)$ & $2(50 \%)$ & $2(50 \%)$ \\
\hline Small cell carcinoma & 0 & 0 & $2(50 \%)$ & 0 & $2(50 \%)$ \\
\hline Suspicious of malignancy & $2(50 \%)$ & $1(25 \%)$ & $3(75 \%)$ & 0 & $1(25 \%)$ \\
\hline Others & $1(25 \%)$ & 0 & $1(25 \%)$ & 0 & 0 \\
\hline
\end{tabular}

Table 2: Shows sensitivity, specificity, positive predictive value, negative predictive Value and Diagnostic Accuracy of all the 4 markers in all the $\mathbf{5 0}$ cases.

\begin{tabular}{|c|c|c|c|c|c|}
\hline Varibales & Sensitivity & Specificity & PPV & PPV & Accuracy \\
\hline CK 5/6 & $100.0 \%$ & $57.1 \%$ & $89.7 \%$ & $100.0 \%$ & $90.9 \%$ \\
\hline TTF-1 & $100.0 \%$ & $96.7 \%$ & $75.0 \%$ & $100.0 \%$ & $97.0 \%$ \\
\hline AE $1 / 3$ & $100.0 \%$ & $0.0 \%$ & $100.0 \%$ & $0.0 \%$ & $100.0 \%$ \\
\hline CK20 & $0.0 \%$ & $100.0 \%$ & $0.0 \%$ & $97.0 \%$ & $97.0 \%$ \\
\hline
\end{tabular}

Table 3: H score

\begin{tabular}{|c|c|c|c|c|c|}
\hline \multicolumn{6}{|c|}{ H score } \\
\hline Diagnosis & & CK 5/6 & TTF-1 & AE1/3 & CK20 \\
\hline \multirow{4}{*}{ Squamous cell carcinoma } & $\mathrm{N}$ & 29 & 28 & 28 & 21 \\
\hline & Mean & 111.379 & 0.000 & 242.536 & 0.000 \\
\hline & SD & 69.7815 & 0.0000 & 38.9876 & 0.0000 \\
\hline & Median & 135.000 & 0.000 & 247.500 & 0.000 \\
\hline \multirow{4}{*}{ Adenocarcinoma } & $\mathrm{N}$ & 4 & 4 & 4 & 3 \\
\hline & Mean & 0.000 & 100.000 & 217.500 & 70.000 \\
\hline & SD & 0.0000 & 70.7107 & 28.7228 & 121.2436 \\
\hline & Median & 0.000 & 125.000 & 225.000 & 0.000 \\
\hline \multirow{4}{*}{ Metastatic Adenocarcinoma } & $\mathrm{N}$ & 1 & 1 & 1 & 1 \\
\hline & Mean & 0.000 & 210.000 & 210.000 & 0.000 \\
\hline & SD & & & & \\
\hline & Median & 0.000 & 210.000 & 210.000 & 0.000 \\
\hline
\end{tabular}




\begin{tabular}{|c|c|c|c|c|c|}
\hline \multicolumn{6}{|c|}{ H score } \\
\hline Diagnosis & & CK 5/6 & TTF-1 & AE1/3 & CK20 \\
\hline \multirow{4}{*}{ Others } & $\mathrm{N}$ & 4 & 4 & 4 & 3 \\
\hline & Mean & 20.000 & 0.000 & 123.750 & 0.000 \\
\hline & SD & 40.0000 & 0.0000 & 62.8987 & 0.0000 \\
\hline & Median & 0.000 & 0.000 & 150.000 & 0.000 \\
\hline \multirow{4}{*}{ Poorly differentiated carcinoma } & $\mathrm{N}$ & 4 & 4 & 4 & 3 \\
\hline & Mean & 45.000 & 0.000 & 208.750 & 140.000 \\
\hline & SD & 90.0000 & 0.0000 & 72.8440 & 121.2436 \\
\hline & Median & 0.000 & 0.000 & 240.000 & 210.000 \\
\hline \multirow{4}{*}{ Small cell carcinoma } & $\mathrm{N}$ & 4 & 4 & 4 & 4 \\
\hline & Mean & 0.000 & 45.000 & 97.500 & 0.000 \\
\hline & SD & 0.0000 & 90.0000 & 113.2475 & 0.0000 \\
\hline & Median & 0.000 & 0.000 & 90.000 & 0.000 \\
\hline \multirow{4}{*}{ Suggestive/Suspicious of Malignancy } & $\mathrm{N}$ & 4 & 4 & 4 & 1 \\
\hline & Mean & 72.500 & 45.000 & 221.250 & 0.000 \\
\hline & SD & 83.8153 & 90.0000 & 33.2603 & \\
\hline & Median & 70.000 & 0.000 & 225.000 & 0.000 \\
\hline
\end{tabular}

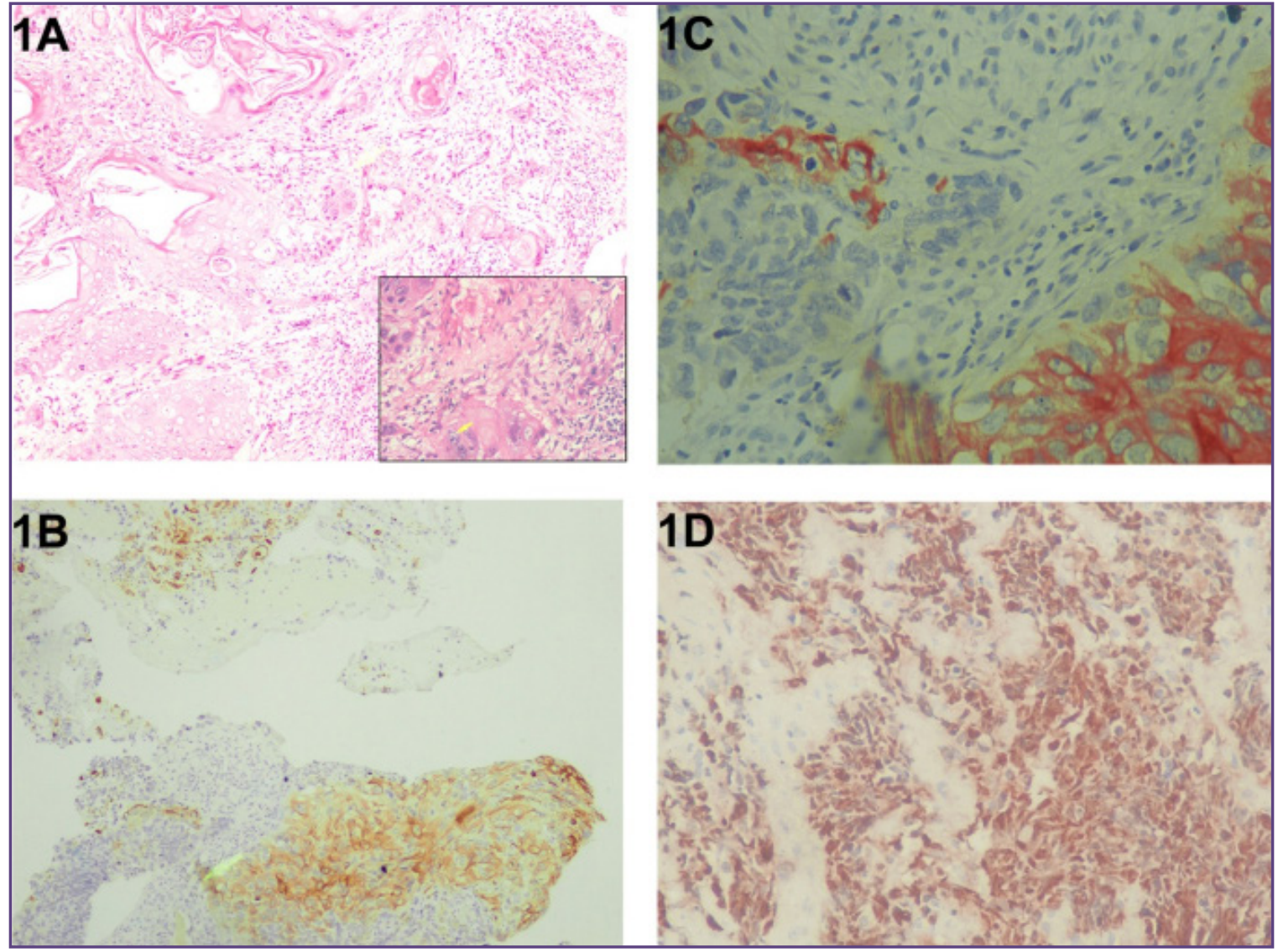

Fig. 1A : Squamous cell carcinoma- Showing tumour cells arranged in sheets and nests ( H\&E stain, 100x) Inset: Showing polygonal tumour cells, having hyperchromatic nuclei \& prominent nucleoli ( H \& E stain , 400x); 1B : Squamous cell carcinoma- Neoplastic cells showing focal cytoplasmic positivity. ( immunostain CK5/6, 100x); 1C: Squamous cell carcinoma - Neoplastic cells showing focal cytoplasmic positivity ( immunostain CK5/6,400x); 1D : Squamous cell carcinoma Neoplastic cells showing strong nuclear positivity ( immunostain Ki-67, 400x). 

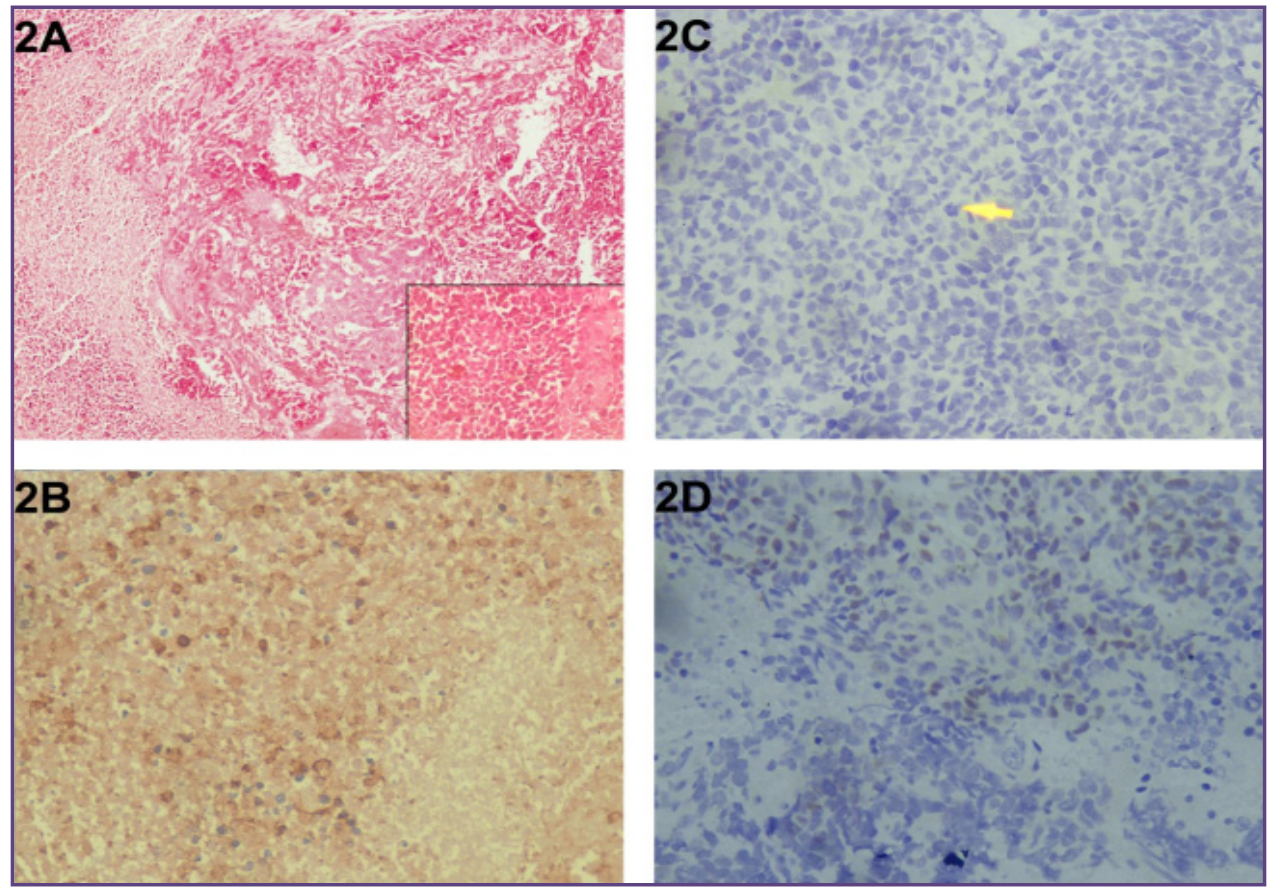

Fig. 2A : Poorly differentiated carcinoma- Showing large areas of necrosis along with a tumour arranged in poorly cohesive sheet ( H\&E stain, 100x) Inset :Showing small to medium sized cells having round to irregular hyperchromatic nuclei ( H\&E stain, 400x); 2B : Poorly differentiated carcinoma - Neoplastic cells showing diffuse cytoplasmic positivity (immunostain CK20, 400x); 2C: Poorly differentiated carcinoma- Shows neoplastic cells negative for immunostain (CK5/6, 400x). ArrowAtypical mitotic figure; 2D: Poorly differentiated carcinoma with ill formed neoplastic glands.

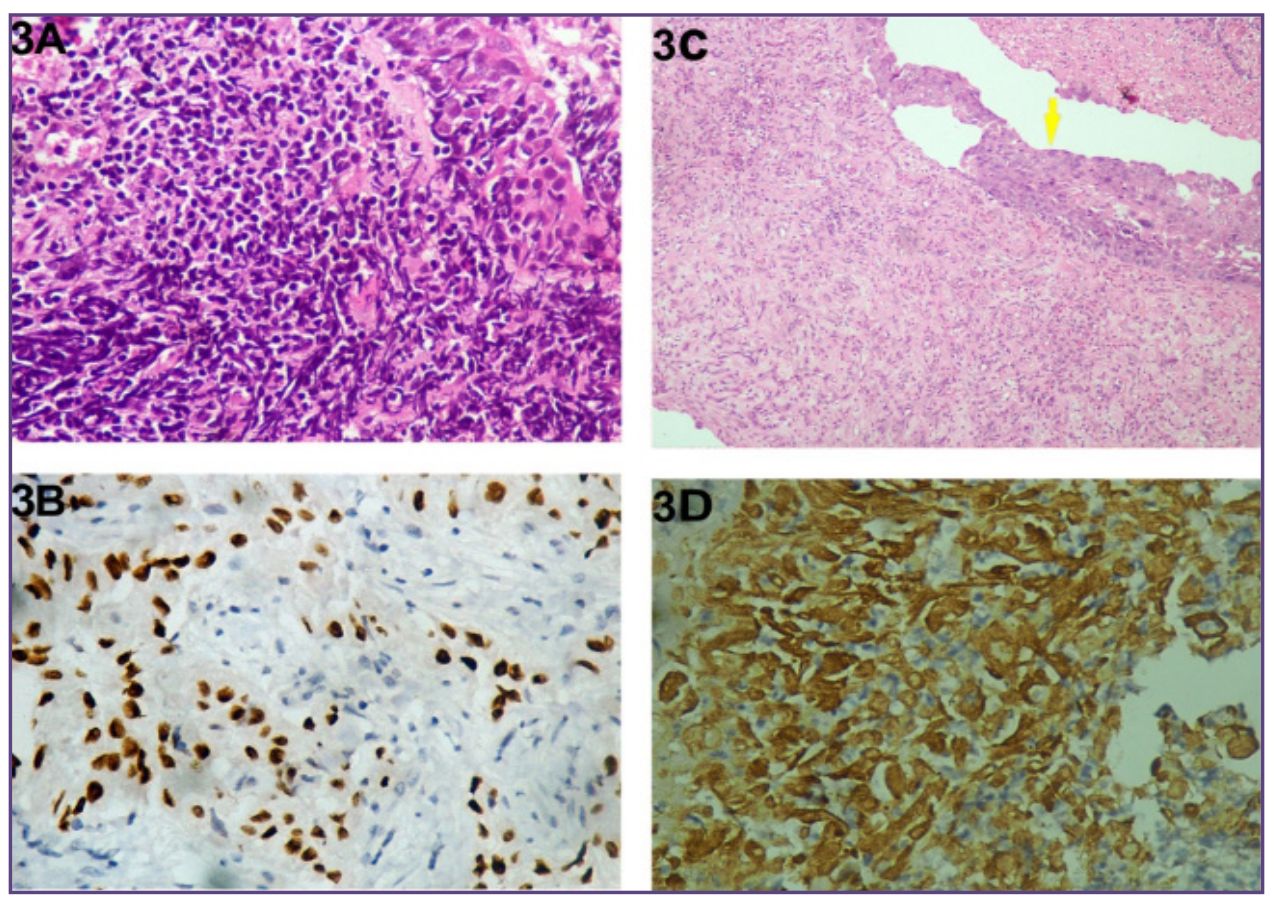

Fig. 3A : Small cell carcinoma- Showing small tumour cells having hyperchromatic nuclei and nuclear moulding(H\&E stain, 100x); 3B: Small cell carcinoma - Neoplastic cells showing strong nuclear positivity (immnostain TTF-1, 400x); 3C: Suspicious of malignancy - Showing small foci of atypical cells (H\&E stain 100x); 3D: Suspicious of malignancy- Neoplastic cells show strong diffuse cytoplasmic positivity for CK5/6 confirming it to be Squmous cell carcinoma ( immunostain CK5/6, 400 x) 
non-squamous cell carcinoma from squamous cell carcinomas is neccessary for effective pulmonary cancer therapy specific for tumor subtype, and thus a number of studies are being undertaken by various institutions with this aim. ${ }^{[12,13]} \mathrm{A}$ combination of IHC panel is effective in reaching a diagnosis, however to date no consensus has been reached for the optimum panel of IHC marker to make a definitive diagnosis of lung carcinoma. Recent studies have advocated the combined use of antibodies for adenocarcinomas (TTF-1 and CK-7) and SCC (p63 and $\mathrm{CK} 5 / 6)$ that provides a very reliable distinction between these 2 NSCLC subtypes. ${ }^{[14]}$ Besides, some of the nonsmall cell lung carcinomas are too poorly differentiated to be accurately subclassified on routine $\mathrm{H} \& \mathrm{E}$ stain, especially if the biopsies are too small. However, recent immunohistochemical studies that focussed on poorly differentiated non-small cell carcinomas in small biopsies when combined with H\&E serve as a gold standard for accurately subclassifying poorly differentiated non-small cell lung carcinomas. ${ }^{[15,16]}$ Besides, TTF-1 and CK20 have ben recently reported to be useful to distinguish between primary and metastatic lung adenocarcinoma.

In this study, we intended to employ a minimum IHC panel that can be used in a tertiary care centre to reach a definite diagnosis in difficult cases. Our study employed the use of four antibodies namely CK5/6, TTF-1, CK20 and AE1/3. Of these CK5/6 and TTF-1 were use to differentiate between Squamous cell carcinoma and Adenocarcinoma in our study.

We observed out of 29 cases of Squamous cell carcinoma diagnosed on H\&E, 26 cases(89.7\%) were positive for CK5/6, thus confirming the diagnosis of SCC on IHC. Whereas, the 3 cases that were diagnosed as Squmous Cell carcinoma on H\&E did not show a positive expression for $\mathrm{CK} 5 / 6$. However, all these cases were positive for AE1/3 which is a pancytokeratin. This erroneous staining pattern may be attributable to an increased crush artefact. All the 29 cases were negative for TTF-1. Thus, similar to other studies, CK5/6 is an excellent marker with certain limitations. ${ }^{[17,18]}$

In our study, out of 5 cases that were diagnosed as adenocarcinoma on $\mathrm{H} \& \mathrm{E}, 4$ cases $(80 \%)$ were positive for TTF-1, thus confirming primary Adenocarcinoma on IHC. One case that was negative for TTF-1, on employing CK 20 showed positive reaction thus confirming it to be Metastatic Adenocarcinoma. All these cases were negative for CK5/6.

TTF-1 is a marker for lung adenocarcinoma and is routinely used in diagnosis of pulmonary adenocarcinoma ${ }^{[19,20]}$ Furthermore, primary and metastatic Adenocarcinoma of lung could be differentiated employing TTF-1 and CK20. In a study by Yue-Chi Su et al, 73\% of primary lung adenocarcinomas expressed TTF-1 staining, whereas all non pumonary adenocarcinomas lacked TTF-1 staining. CK 20 expression was significantly more prevalent in metastatic adenocarcinomas. ${ }^{[16]}$ They concluded that a combination panel could distinguish between pulmonary and extrapulmonary adenocarcinomas metastatic to lung.

It is interesting that use of IHC did not improve diagnostic accuracy for SCC in our study which is a finding similar to Ocque $\mathrm{R}$ et al . ${ }^{[21]}$ In another study by Jorda et al, application of IHC markers increased the yield of detection of SCC from $35 \%$ to $88 \% .{ }^{[22]}$ The distinction in experiences with various studies may be attributable to the type of antibody used, amount of study material and experience of pathologist.

However, increased use of IHC markers as ancillary method was found to increase the interpretation and classification for adenocarcinomas as primary or metastatic. This is further supported by studies by other authors. ${ }^{[16-22]}$ TTF-1 is a marker of lung adenocarcinoma and is routinely used in the diagnosis of pulmonary adenocarcinoma and this has been demonstrated in a number of studies. ${ }^{[23]}$

Out of 4 cases diagnosed as Small cell carcinoma on H\&E, only 1 case $(25 \%)$ could be confirmed to be Small cell carcinoma exhibiting both AE1/3 and TTF-1 positivity. The remaining 3 cases did not show a positive staining and may be attributable to small size of biopsy, crush artefact and observer variation. Our observation was in contrast to findings in a study by Miyauchi et al who observed TTF1 immunoreactivity in 79 out of 96 cases $(82 \%) \cdot{ }^{[24]}$ This may be attributable to small sample size of Small Cell carcinoma cases in our study.

Out of 4 cases diagnosed as Poorly differentiated carcinma on $\mathrm{H} \& \mathrm{E}, 3$ cases could be further confirmed and diagnosed employing IHC markers. One case $(25 \%)$ was confirmed to be Squamous cell Carcinoma exhibiting positive CK5/6 expression and other 2 cases exhibited positive CK20 expression $(50 \%)$ thus confirming it to be metastatic adenocarcinoma. Thus the antibodies employed in present study were able to diagnose Poorly differentiated cases of lung carcinoma. Limited tumour size may be one of the factor for non- conformation of one case in our study.

In the category of Suspicious/Suggestive of malignancy on H\&E included the cases that could not be definitely labelled as malignant due to small size of sample and features that fall short of malignancy. For 3 cases a confirmed diagnosis could be given on employing IHC markers. Of these 3 cases, 2 cases were confirmed to be 
Squamous cell carcinoma exhibiting a positive expression for CK5/6 and one case exhibited a positive expression for TTF-1 thus confirming it to be primary adenocarcinoma. These 3 cases also exhibited a positive expression for $\mathrm{AE} 1 / 3$. One case that remained inconclusive even after applying IHC markers was asked for follow up and repeat biopsy. Thus, limitation of size of tumour poses difficulty in interpretation even on IHC.

The biopsies that showed epithelial dysplasia were grouped under the category of Others in our study and this group included 4 cases. However, positive expression of AE1/3 and CK5/6 was seen in only 1 case which confirmed it to be Squamous cell carcinoma. The remaining 3 cases in this category were negative for AE1/3, CK20, CK5/6 and TTF-1.

For squamous cell carcinoma, CK5/6 showed a sensitivity and specificiy of $100 \%$ and $57.1 \%$ respectively while TTF1 showed a sensitivity and specificity of $100 \%$ and $96.7 \%$ respectively in our study. In a study by Kusum Kapila et al CK5/6 showed a sensitivity and specificity of $53 \%$ and $96 \%$ respectively and TTF- 1 was found to be most specific marker for adenocarcinoma with $60 \%$ sensitivity and $98 \%$ specificity.[25]

Another study by Gregorz T Gurda et al in 72 cases of primary adencarcinoma TTF- 1 showed a sensitivity and specificity of $84.5 \%$ and $96.4 \%$ respectively. In 30 cases of squamous cell carcinomaCK $5 / 6$ showed a sensitivity and specificity of $100 \%$ and $77.8 \%$ respectively ${ }^{[26]}$. Thus, our study is closely related with studies by other authors in India and West in respect to sensitivity and specificity of TTF-1 and CK5/6

\section{Conclusion}

In conclusion, our study showed that most of the lung tumours could be diagnosed morphologically if the biopsy is adequate. Use of IHC in poorly differentiated tumours help us to reach a definite diagnosis which is otherwise not possible on $\mathrm{H} \& \mathrm{E}$.

After the development of widely available and applicable monoclonal antibodies, identification of immunophenotypic profile for most of the lung tumours is now possible. Definitive diagnosis on employing IHC is important for our clinical colleagues for better distinction between adenocarcinoma and SCC management.

Thus, using IHC either singly or a panel is a useful adjunct to morphological features and clinical parameters in the diagnosis and management of lung tumours. However, ancillary studies should not be used as additional diagnostic confirmation in which morphology itself is sufficient in subtyping of NSCLC.

\section{References}

1. Edges SB, Compton CC. The American Joint Committee on Cancer: The 7th edition of the AJCC cancer staging manual and the future of TNM. Ann Surg Oncol 2010;17(6):1471-4.

2. Siegel R, Ma J, Zou Z, Jemal A. Cancer statistics,2014.CA: a cancer journal for clinicians 2014;64(1):9-29.

3. Indian council of Medical Research;2013. National Cancer Registry Programme. Three year report of Population Based Cancer Registries:2009-11.

4. Travis WD, Brambilla E, Noguchi M, Nicholson AG, Geising $\mathrm{KR}$, Yatabe $\mathrm{Y}$ et al. International association for the study of lung cancer/American thoracic society/ European respiratory society international multidisciplinary classification of lung adenocarcinoma. J Thorac Oncol 2011;6(2):244-85.

5. Travis WD, Harris Cc. Pathology and genetics of tumours of the lung, pleura, thymus and heart. Feance: IARC Press, 2004;2004.

6. Grilley-Olson JE, Hayes DN, Moore DT, Lesile Ko, Wilkerson MD, Qaqish BF et al. Validation of Interobserver Agreement in Lung Cancer Assesment: Hematoxylin-Eosin Diagnostic Reproducibility for Non- Small Cell Lung Cancer: The 2004World Health Organisation Classification and Therapeutically Relevant Subsets. Arch Pathol Lab Med 2012; 137(1):32-40.

7. Bishop JA, Sharma R, Illegi PB. Napsin A and thyroid transcription factor-1 expression in carcinomas of the lung, breast, pancreas, colon, kidney, thyroid and malignant mesothelioma. Hum Pathol. 2010;4191):20-25.

8. Carvalho L. Reclassifying bronchial-pulmonary carcinoma: differentiating histological type in biopsies by immunohistochemistry. Revista Portuguesa Pneumologia (English Edition)2009; 15(6):1101-19.

9. Munfus-McCray D, Cui M, Zhang Z, Gabrielson Askin F, Li QK. Comparison of EGFR and KRAS mutations in primary and unpaired metastatic lung adenocarcinoma with potential chemotherapy effect. Hum Pathol 2013;44(7):1286-92.

10. Yang P,Allen MS, Aubry MC, Wampfler JA, Marks RS, Edell ES, Thibodeau S, Adjeji AA et al. Clinical features of 5,628 primary lung cancer patients: experience at Mayo Clinic from 1997 to 2003. CHEST Journal. 2005;128(1):452-62.

11. Rekhtman N, Ang DC, Sima CS, Travis WD, Moreira AL. Immunohistochemical algorithm for differentiation of lung adenocarcinoma and squamous cell carcinoma based on large series of whole-tissue sections with validation in small specimens . Modern Pathology. 2011;24(10):1348

12. Whithaus K, Fukuoka J, Prihoda TJ, Jagirdar J: Evaluation of napsin A, Cytokeratin 5/6, p63 and thyroid transcription factor 1 in adenocarcinoma versus squamous cell carcinoma of the lung. Arch Pathol Lab Med.136:155-62,2012.

13. Yanagita E, Imagawa $\mathrm{N}$, Ohbayaschi $\mathrm{C}$, Itoh $\mathrm{T}$ : Rapid multiplex immunohistochemistry using the 4-antibody cocktail YANA-4in differentiating primary adenocarcinoma from squamous cell carcinoma of the lung. Appl. Immunohistochem Mol Morphol. 19 :509-13;2011. 
14. Rossi G, Pelosi G, Giaziano Pet al. A reevaluation of the clinical significance of histological subtyping of non-small cell lung carcinomas: diagnostic algorithms in the era of personalized treatments. Int J Surg Pathol. 2009;17:206-18.

15. Jagirdar J. Application of immunohistochemistry to the diagnosis of primary and metastatic carcinoma to the lung. Arch. Pathol Lab Med 2008;132(3):384-96.

16. Su YC, Hsu YC, Chai CY. Role f TTF-1, CK-20 and CK7 Immunohistochemistry for diagnosis of primary and secondary lung adenocarcinoma. Kaohsung $\mathrm{J}$ Med Sci.2006;22(1):14-9.

17. Kangi A, Gurel D, Tuna B. The diagnostic value of TTf1, CK5/6, and p63 immunostaining in classification of lung carcinomas. Appl Immunohistochem Mol. Morphol.2007;15:415-20.

18. Khayyata S, Yun S, Pasha $\mathrm{T}$ et al. Value of p63 and CK5/6 in distinguishing squamous cell carcinoma from adenocarcinoma in lung fine-needle aspiration specimens. Diagn Cytopathol.2009;37:178-83.

19. Ng WK, Chow JC, Ng PK: Thyroid transcription factor-1 is highly sensitive and specific in differentiating metastatic pulmonary from extrapulmonary adenocarcinoma in effusion cytology specimens. Cancer 2002;96:43-48.

20. Yatabe Y, Mitsudomi J, TakahashiT: TTF-1 expression in pulmonary adenocarcinoma. Am J Surg Pathol.2002;26:763-73.

21. Ocque R, Tochigi N, Ohori P, Dacie S et al. Usefulness of Immunohistochemical and Histochemical Studies in classification of lung adenocarcinoma and Squamous cell carcinoma in Cytologic Specimens. Am J Clin Pathol.2016;136:81-87.

22. Jorda H, Gomez-Fernandez C, Garcia $M$ et al. p63 differentiates subtypes of non small-cell carcinoma of lung in cytologic samples: implications in treatment selection. Cancer 2009:117;46-50.

23. Shibuki Y, Tsula K, Nomoto K et al: Immunocytochemical study of specific immunohistochemical markers for primary lung. J Jpn Clin Cytol2007; 46:120-21.

24. Miyarchi E, Motoi N, Ono H, Ninomya h, Ohyaanay F et al. Distinct charactersticks of small cell lung cancer correlate with central or peripheral origin: subtyping basal on location and expression of transcription factor TTF-1. Medicine 2015, 94(51).....

25. Kapila K, Al-Ayadhy B, Francis IM, George SS, AlJassar A. Subclassification of pulmonary non-small cell lung carcinoma in fine needle aspirates using a limited immunohistochemistry panel. J Cytol. 2013 Oct-Dec; 30(4): $223-225$

26. Gurda GT, Zhang L, Wang Y, Chen L, Geddes S, Cho WC et al. Utility of five commonly used immunohistochemical markers TTF-1< Napsin A, CK7, CK5/6 and p63 in primary and metastatic adenocarcinoma and squamous cell carcinoma of the lung: a retrospective study of 246 fine needle aspiration cases. Clinical and translational medicine.2015Apr21;4(1):16.

*Corresponding author:

Dr. Aparna Bhardwaj, 1, main Street, ashirwad enclave, Chakrata Road, Dehradun, 248001. India

Phone: +91 9411718270

Email: aparnapande1977@gmail.com

Financial or other Competing Interests: None. 